\title{
Robust Distributed Noise Reduction in Hearing Aids with External Acoustic Sensor Nodes
}

\author{
Alexander Bertrand and Marc Moonen (EURASIP Member) \\ Department of Electrical Engineering (ESAT-SCD), Katholieke Universiteit Leuven, Kasteelpark Arenberg 10, \\ 3001 Leuven, Belgium \\ Correspondence should be addressed to Alexander Bertrand, alexander.bertrand@esat.kuleuven.be
}

Received 15 December 2008; Revised 17 June 2009; Accepted 24 August 2009

Recommended by Walter Kellermann

The benefit of using external acoustic sensor nodes for noise reduction in hearing aids is demonstrated in a simulated acoustic scenario with multiple sound sources. A distributed adaptive node-specific signal estimation (DANSE) algorithm, that has a reduced communication bandwidth and computational load, is evaluated. Batch-mode simulations compare the noise reduction performance of a centralized multi-channel Wiener filter (MWF) with DANSE. In the simulated scenario, DANSE is observed not to be able to achieve the same performance as its centralized MWF equivalent, although in theory both should generate the same set of filters. A modification to DANSE is proposed to increase its robustness, yielding smaller discrepancy between the performance of DANSE and the centralized MWF. Furthermore, the influence of several parameters such as the DFT size used for frequency domain processing and possible delays in the communication link between nodes is investigated.

Copyright (C) 2009 A. Bertrand and M. Moonen. This is an open access article distributed under the Creative Commons Attribution License, which permits unrestricted use, distribution, and reproduction in any medium, provided the original work is properly cited.

\section{Introduction}

Noise reduction algorithms are crucial in hearing aids to improve speech understanding in background noise. For every increase of $1 \mathrm{~dB}$ in signal-to-noise ratio (SNR), speech understanding increases by roughly $10 \%[1]$. By using an array of microphones, it is possible to exploit spatial characteristics of the acoustic scenario. However, in many classical beamforming applications, the acoustic field is sampled only locally because the microphones are placed close to each other. The noise reduction performance can often be increased when extra microphones are used at significantly different positions in the acoustic field. For example, an exchange of microphone signals between a pair of hearing aids in a binaural configuration, that is, one at each ear, can significantly improve the noise reduction performance [2-11]. The distribution of extra acoustic sensor nodes in the acoustic environment, each having a signal processing unit and a wireless link, allows further performance improvement. For instance, small sensor nodes can be incorporated into clothing, or placed strategically either close to desired sources to obtain high SNR signals, or close to noise sources to collect noise references. In a scenario with multiple hearing aid users, the different hearing aids can exchange signals to improve their performance through cooperation.

The setup envisaged here requires a wireless link between the hearing aid and the supporting external acoustic sensor nodes. A distributed approach using compressed signals is needed, since collecting and processing all available microphone signals at the hearing aid itself would require a large communication bandwidth and computational power. Furthermore, since the positions of the external nodes are unknown, the algorithm should be adaptive and able to cope with unknown microphone positions. Therefore, a multichannel Wiener filter (MWF) approach is considered, since an MWF estimates the clean speech signal without relying on prior knowledge on the microphone positions [12]. In [13, 14], a distributed adaptive node-specific signal estimation (DANSE) algorithm is introduced for linear MMSE signal 
estimation in a sensor network, which significantly reduces the communication bandwidth while still obtaining the optimal linear estimators, that is, the Wiener filters, as if each node has access to all signals in the network. The term "node-specific" refers to the scenario in which each node acts as a data-sink and estimates a different desired signal. This situation is particularly interesting in the context of noise reduction in binaural hearing aids where the two hearing aids estimate differently filtered versions of the same desired speech source signal, which is indeed important to preserve the auditory cues for directional hearing [15-18]. In [19], a pruned version of the DANSE algorithm, referred to as distributed multichannel Wiener filtering (db-MWF), has been used for binaural noise reduction. In the case of a single desired source signal, it was proven that db-MWF converges to the optimal all-microphone Wiener filter settings in both hearing aids. The more general DANSE algorithm allows the incorporation of multiple desired sources and more than two nodes. Furthermore, it allows for uncoordinated updating where each node decides independently in which iteration steps it updates its parameters, possibly simultaneously with other nodes [20]. This in particular avoids the need for a network wide protocol that coordinates the updates between nodes.

In this paper, batch-mode simulation results are described to demonstrate the benefit of using additional external sensor nodes for noise reduction in hearing aids. Furthermore, the DANSE algorithm is reformulated in a noise reduction context, and a batch-mode analysis of the noise reduction performance of DANSE is provided. The results are compared to those obtained with the centralized MWF algorithm that has access to all signals in the network to compute the optimal Wiener filters. Although in theory the DANSE algorithm converges to the same filters as the centralized MWF algorithm, this is not the case in the simulated scenario. The resulting decrease in performance is explained and a modified algorithm is then proposed to increase robustness and to allow the algorithm to converge to the same filters as in the centralized MWF algorithm. Furthermore, the effectiveness of relaxation is shown when nodes update their filters simultaneously, as well as the influence of several parameters such as the DFT size used for frequency domain processing, and possible delays within the communication link. The simulations in this paper show the potential of DANSE for noise reduction, as suggested in $[13,14]$, and provide a proof-of-concept for applying the algorithm in cooperative acoustic sensor networks for distributed noise reduction applications, such as hearing aids.

The outline of this paper is as follows. In Section 2, the data model is introduced and the multi-channel Wiener filtering process is reviewed. In Section 3, a description of the simulated acoustic scenario is provided. Moreover, an analysis of the benefits achieved using external acoustic sensor nodes is given. In Section 4, the DANSE algorithm is reviewed in the context of noise reduction. A modification to DANSE increasing robustness is introduced in Section 5. Batch-mode simulation results are given in Section 6. Since some practical aspects are disregarded in the simulations, some remarks and open problems concerning a practical implementation of the algorithm are given in Section 7.

\section{Data Model and Multichannel Wiener Filtering}

2.1. Data Model and Notation. A general fully connected broadcasting sensor network with $J$ nodes is considered, in which each node $k$ has direct access to a specific set of $M_{k}$ microphones, with $M=\sum_{k=1}^{J} M_{k}$ (see Figure 1). Nodes can be either a hearing aid or a supporting external acoustic sensor node. Each microphone signal $m$ of node $k$ can be described in the frequency domain as

$$
y_{k m}(\omega)=x_{k m}(\omega)+v_{k m}(\omega), \quad m=1, \ldots, M_{k},
$$

where $x_{k m}(\omega)$ is a desired speech component and $v_{k m}(\omega)$ an undesired noise component. Although $x_{k m}(\omega)$ is referred to as the desired speech component, $v_{k m}(\omega)$ is not necessarily nonspeech, that is, undesired speech sources may be included in $v_{k m}(\omega)$. All subsequent algorithms will be implemented in the frequency domain, where (1) is approximated based on finite-length time-to-frequency domain transformations. For conciseness, the frequency-domain variable $\omega$ will be omitted. All signals $y_{k m}$ of node $k$ are stacked in an $M_{k}$ dimensional vector $\mathbf{y}_{k}$, and all vectors $\mathbf{y}_{k}$ are stacked in an $M$-dimensional vector $\mathbf{y}$. The vectors $\mathbf{x}_{k}, \mathbf{v}_{k}$ and $\mathbf{x}, \mathbf{v}$ are similarly constructed. The network-wide data model can now be written as $\mathbf{y}=\mathbf{x}+\mathbf{v}$. Notice that the desired speech component $\mathbf{x}$ may consist of multiple desired source signals, for example when a hearing aid user is listening to a conversation between multiple speakers, possibly talking simultaneously. If there are $Q$ desired speech sources, then

$$
\mathbf{x}=\text { As, }
$$

where $\mathbf{A}$ is an $M \times Q$-dimensional steering matrix and $\mathbf{s}$ a $Q$-dimensional vector containing the $Q$ desired sources. Matrix A contains the acoustic transfer functions (evaluated at frequency $\omega$ ) from each of the speech sources to all microphones, incorporating room acoustics and microphone characteristics.

2.2. Centralized Multichannel Wiener Filtering. The goal of each node $k$ is to estimate the desired speech component $x_{k m}$ in its $m$ th microphone, selected to be the reference microphone. Without loss of generality, it is assumed that the reference microphone always corresponds to $m=1$. For the time being, it is assumed that each node has access to all microphone signals in the network. Node $k$ then performs a filter-and-sum operation on the microphone signals with filter coefficients $\mathbf{w}_{k}$ that minimize the following MSE cost function:

$$
J_{k}\left(\mathbf{w}_{k}\right)=E\left\{\left|x_{k 1}-\mathbf{w}_{k}^{H} \mathbf{y}\right|^{2}\right\},
$$

where $E\{\cdot\}$ denotes the expected value operator, and where the superscript $H$ denotes the conjugate transpose operator. 


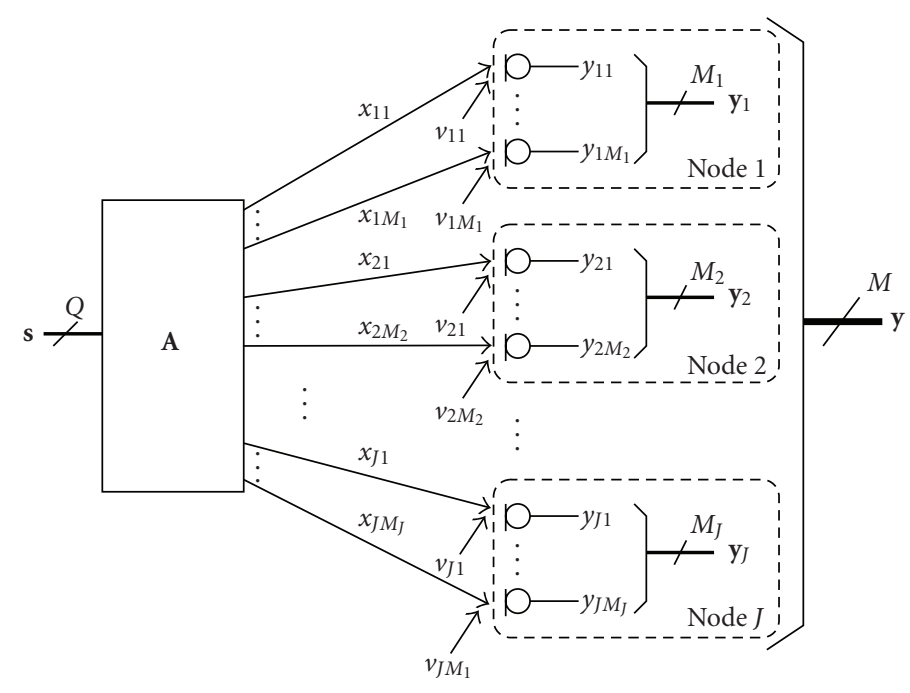

FIGURE 1: Data model for a sensor network with $J$ sensor nodes, in which node $k$ collects $M_{k}$ noisy observations of the $Q$ source signals in s.

Notice that at each node $k$, one such MSE problem is to be solved for each frequency bin. The minimum of (3) corresponds to the well-known Wiener filter solution:

$$
\widehat{\mathbf{w}}_{k}=\mathbf{R}_{y y}^{-1} \mathbf{R}_{y x} \mathbf{e}_{k 1},
$$

with $\mathbf{R}_{y y}=E\left\{\mathbf{y} \mathbf{y}^{H}\right\}, \mathbf{R}_{y x}=E\left\{\mathbf{y} \mathbf{x}^{H}\right\}$, and $\mathbf{e}_{k 1}$ being an $M$ dimensional vector with only one entry equal to 1 and all other entries equal to 0 , which selects the column of $\mathbf{R}_{y x}$ corresponding to the reference microphone of node $k$. This procedure is referred to as multi-channel Wiener filtering (MWF). If the desired speech sources are uncorrelated to the noise, then $\mathbf{R}_{y x}=\mathbf{R}_{x x}=E\left\{\mathbf{x x}^{H}\right\}$. In the remaining of this paper, it is implicitly assumed that all $Q$ desired sources may be active at the same time, yielding a rank- $Q$ speech correlation matrix $\mathbf{R}_{x x}$. In practice, $\mathbf{R}_{x x}$ is unknown, but can be estimated from

$$
\mathbf{R}_{x x}=\mathbf{R}_{y y}-\mathbf{R}_{v v}
$$

with $\mathbf{R}_{v v}=E\left\{\mathbf{v} \mathbf{v}^{H}\right\}$. The noise correlation matrix $\mathbf{R}_{v v}$ can be (re-)estimated during noise-only periods and $\mathbf{R}_{y y}$ can be (re-)estimated during speech-and-noise periods, requiring a voice activity detection (VAD) mechanism. Even when the noise sources and the speech source are not stationary, these practical estimators are found to yield good noise reduction performance $[15,19]$.

\section{Simulation Scenario and the Benefit of External Acoustic Sensor Nodes}

The performance of microphone array based noise reduction typically increases with the number of microphones. However, the number of microphones that can be placed on a hearing aid is limited, and the acoustic field is only sampled locally, that is, at the hearing aid itself. Therefore, there is often a large distance between the location of the desired source and the microphone array, which results in signals with low SNR. In fact, the SNR decreases with $6 \mathrm{~dB}$ for every doubling of the distance between a source and a microphone. The noise reduction performance can therefore be greatly increased by using supporting external acoustic sensor nodes that are connected to the hearing aid through a wireless link.

To assess the potential improvement that can be obtained by adding external sensor nodes, a multi-source scenario is simulated using the image method [21]. Figure 2 shows a schematic illustration of the scenario. The room is cubical $(5 \mathrm{~m} \times 5 \mathrm{~m} \times 5 \mathrm{~m})$ with a reflection coefficient of 0.4 at the floor, the ceiling and at every wall. According to Sabine's formula this corresponds to a reverberation time of $T_{60}=$ $0.222 \mathrm{~s}$. There are two hearing aid users listening to speaker $\mathrm{C}$, who produces a desired speech signal. One hearing aid user has 2 hearing aids (node 2 and 3 ) and the other has one hearing aid at the right ear (node 4). All hearing aids have three omnidirectional microphones with a spacing of $1 \mathrm{~cm}$. Head shadow effects are not taken into account. Node 1 is an external microphone array containing six omnidirectional microphones placed $2 \mathrm{~cm}$ from each other. Speakers A and B both produce speech signals interfering with speaker C. All speech signals are sentences from the HINT (Hearing in Noise Test) database [22]. The upper left loudspeaker produces multi-talker babble noise (Auditec) with a power normalized to obtain an input broadband SNR of $0 \mathrm{~dB}$ in the first microphone of node 4, which is used as the reference node. In addition to the localized noise sources, all microphone signals have an uncorrelated noise component which consist of white noise with power that is $10 \%$ of the power of the desired signal in the first microphone of node 4. All nodes and all sound sources are in the same horizontal plane, $2 \mathrm{~m}$ above ground level.

Notice that this is a difficult scenario, with many sources and highly non-stationary (speech) noise. This kind of scenario brings many practical issues, especially with respect to reliable VAD decisions (cf. Section 7). Throughout this paper, many of these practical aspects are disregarded. The aim here is to demonstrate the benefit that can be achieved 


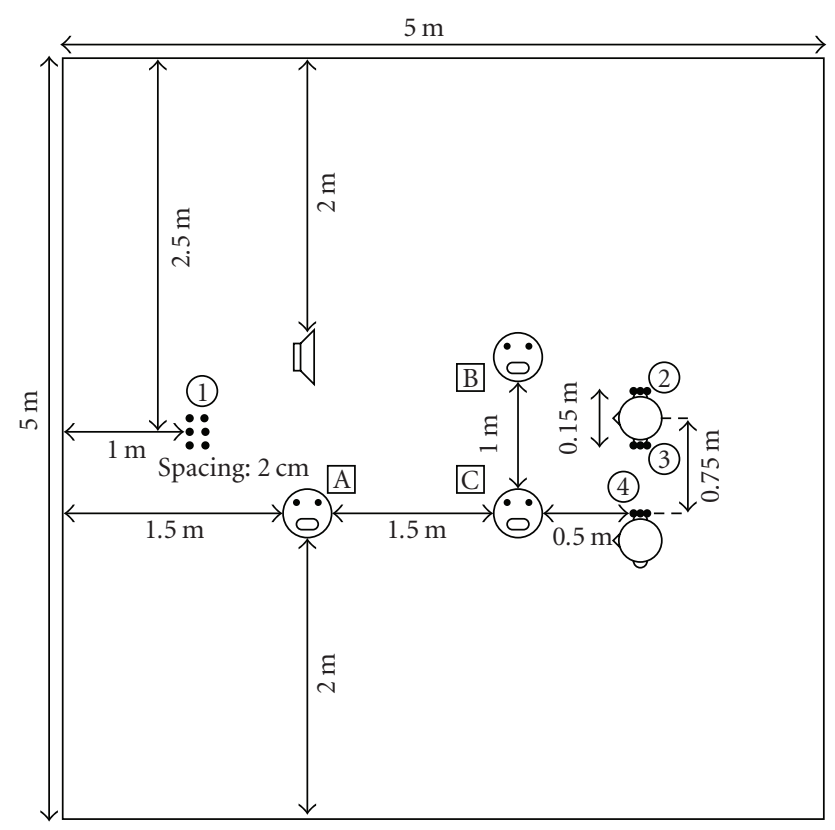

FIgURE 2: The acoustic scenario used in the simulations throughout this paper. Two persons with hearing aids are listening to speaker C. The other sources produce interference noise.

with external sensor nodes, in particular in multi-source scenarios. Furthermore, the theoretical performance of the DANSE algorithm, introduced in Section 4, will be assessed with respect to the centralized MWF algorithm. To isolate the effects of VAD errors and estimation errors on the correlation matrices, all experiments are performed in batch mode with ideal VADs.

Two performance measures are used to assess the quality of the noise reduction algorithms, namely the broadband signal-to-noise ratio (SNR) and the signal-to-distortion ratio (SDR). The SNR and SDR at node $k$ are defined as

$$
\begin{aligned}
& \mathrm{SNR}=10 \log _{10} \frac{E\left\{\hat{x}_{k}[t]^{2}\right\}}{E\left\{\hat{n}_{k}[t]^{2}\right\}}, \\
& \mathrm{SDR}=10 \log _{10} \frac{E\left\{x_{k 1}[t]^{2}\right\}}{E\left\{\left(x_{k 1}[t]-\hat{x}_{k}[t]\right)^{2}\right\}}
\end{aligned}
$$

with $\hat{n}_{k}[t]$ and $\hat{x}_{k}[t]$ the time domain noise component and the desired speech component respectively at the output at node $k$, and $x_{k 1}[t]$ the desired time domain speech component in the reference microphone of node $k$.

The sampling frequency is $32 \mathrm{kHz}$ in all experiments. The frequency domain noise reduction is based on DFT's with size equal to $L=512$ if not specified otherwise. Notice that $L$ is equivalent to the filter length of the time domain filters that are implicitly applied to the microphone signals. The DFT size $L=512$ is relatively large, which is due to the fact that microphones are far apart from each other, leading to higher time differences of arrival (TDOA) demanding longer filters to exploit spatial information. If the filter lengths are too short to allow a sufficient alignment between the signals, then the noise reduction performance degrades. This is evaluated in Section 6.4. To allow small DFT-sizes, yet large distances between microphones, delay compensation should be introduced in the local microphone signals or the received signals at each node. However, since hearing aids typically have hard constraints on the processing delay to maintain lip synchronization, this delay compensation is restricted. This, in effect, introduces a trade-off between input-output delay and noise reduction performance.

Figure 3(a) shows the output SNR and SDR of the centralized MWF procedure at node 4 when five different subsets of microphones are used for the noise reduction:

(1) the microphone signals of node 4 itself;

(2) the microphone signals of node 1 in addition to the microphone signals of node 4 itself;

(3) the microphone signals of node 2 in addition to the microphone signals of node 4 itself;

(4) the first microphone signal at every node in addition to all microphone signals of node 4 itself; this is equivalent to a scenario where the network supporting node 4 consists of single-microphone nodes, that is, $M_{k}=1$, for $k=1, \ldots, 3$;

(5) all microphone signals in the network.

The benefit of adding external microphones is very clear in this graph. It also shows that microphones with a significantly different position contribute more than microphones that are closely spaced. Indeed, Cases 2, 3 and 4 both add three extra microphone signals, but the benefit is largest in Case 4 , in which the additional microphones are relatively set far apart. However, using multi-microphone nodes (Case 5) still produces a significant benefit of about $25 \%(2 \mathrm{~dB})$ in comparison to single-microphone nodes (Case 4). Notice that the benefit of placing external microphones, and the benefit of using multi-microphone nodes in comparison to single-microphone nodes, is of course very scenario specific. For instance, if the vertical position of node 1 is reduced by $0.5 \mathrm{~m}$ in Figure 2, then the difference between singlemicrophone nodes (Case 4) and multi-microphone nodes (Case 5 ) is more than $3 \mathrm{~dB}$, as shown in Figure 3(b), which correponds to an improvement of almost 50\%.

\section{The DANSE Algorithm}

In Section 3, simulations showed that adding external microphones in addition to the microphones available in a hearing aid may yield a great benefit in terms of both noise suppression and speech distortion. Not surprisingly, adding external nodes with multiple microphones boosts the performance even more. However, the latter introduces a significant increase in communication bandwidth, depending on the number of microphones in each node. Furthermore, the dimensions of the correlation matrix to be inverted in formula (4) may grow significantly. However, if each node has its own signal processor unit, this extra communication bandwidth can be reduced and the computation can be distributed by using the distributed adaptive node-specific 

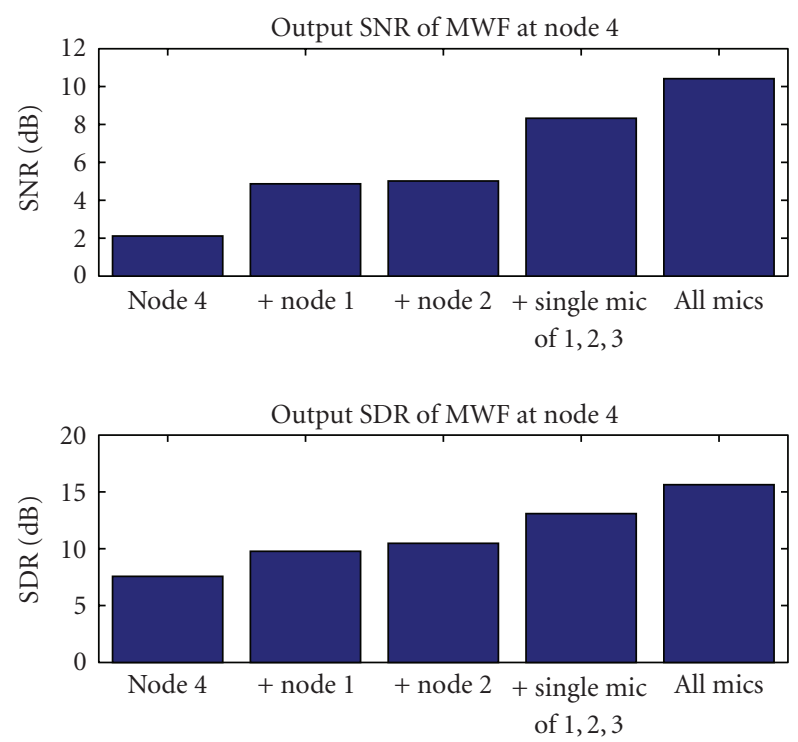

(a) Scenario of Figure 2
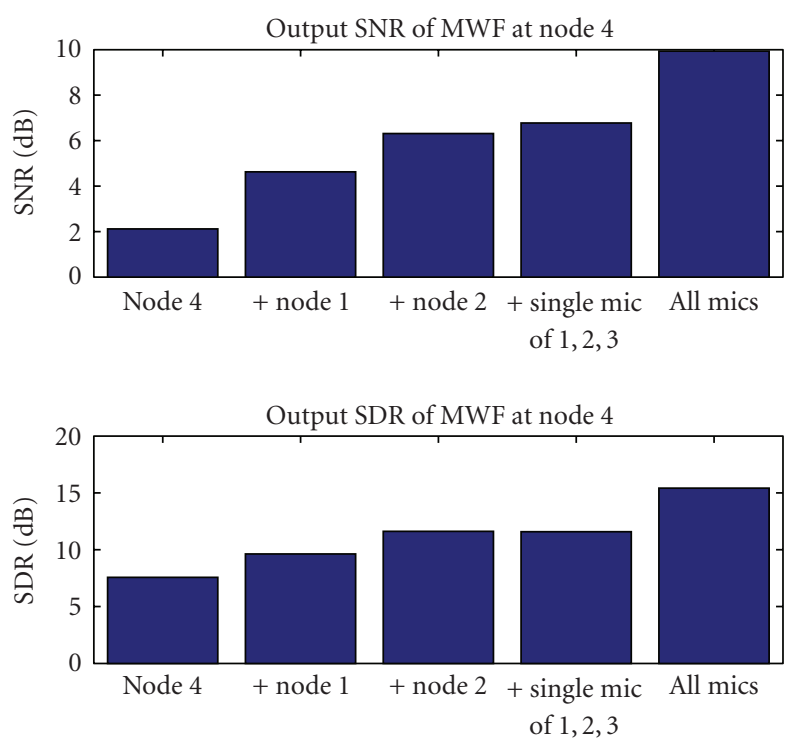

(b) Scenario of Figure 2 with vertical position of node 1 reduced by $0.5 \mathrm{~m}$

FIGURE 3: Comparison of output SNR and SDR of MWF at node 4 for five different microphone subsets.

signal estimation (DANSE) algorithm, as proposed in [13, 14]. The DANSE algorithm computes the optimal network wide Wiener filter in a distributed, iterative fashion. In this section this algorithm is briefly reviewed and reformulated in a noise reduction context.

4.1. The DANSE $E_{K}$ Algorithm. In the $\mathrm{DANSE}_{K}$ algorithm, each node $k$ estimates $K$ different desired signals, corresponding to the desired speech components in $K$ of its microphones (assuming that $K \leq M_{k}, \forall k \in\{1, \ldots, J\}$ ). Without loss of generality, it is assumed that the first $K$ microphones are selected, that is, the signal to be estimated is the $K$-channel signal $\overline{\mathbf{x}}_{k}=\left[x_{k 1} \cdots x_{k K}\right]^{T}$. The first entry in this vector corresponds to the reference microphone, whereas the other $K-1$ entries should be viewed as auxiliary channels. They are required to fully capture the signal subspace spanned by the desired source signals. Indeed, if $K$ is chosen equal to $Q$, the $K$ channels of $\overline{\mathbf{x}}_{k}$ define the same signal subspace as defined by the channels in $\mathbf{s}$, that is,

$$
\overline{\mathbf{x}}_{k}=\mathbf{A}_{k} \mathbf{s}
$$

where $\mathbf{A}_{k}$ denotes a $K \times K$ submatrix of the steering matrix A in formula (2). $K$ being equal to $Q$ is a requirement for DANSE $_{K}$ to be equivalent to the centralized MWF solution (see Theorem 1). The case in which $K \neq Q$ is not considered here. For a more detailed discussion why these auxiliary channels are introduced, we refer to [13].

Each node $k$ estimates its desired signal $\overline{\mathbf{x}}_{k}$ with respect to a corresponding MSE cost function

$$
J_{k}\left(\mathbf{W}_{k}\right)=E\left\{\left\|\overline{\mathbf{x}}_{k}-\mathbf{W}_{k}^{H} \mathbf{y}\right\|^{2}\right\}
$$

with $\mathbf{W}_{k}$ an $M \times K$ matrix, defining a multiple-input multiple-output (MIMO) filter. Notice that this corresponds to $K$ independent estimation problems in which the same $M$ channel input signal $\mathbf{y}$ is used. Similarly to (3), the Wiener solution of (9) is given by

$$
\widehat{\mathbf{W}}_{k}=\mathbf{R}_{y y}^{-1} \mathbf{R}_{x x} \mathbf{E}_{k}
$$

with

$$
\mathbf{E}_{k}=\left[\begin{array}{c}
\mathbf{I}_{K} \\
\mathbf{O}_{(M-K) \times K}
\end{array}\right]
$$

with $\mathbf{I}_{K}$ denoting the $K \times K$ identity matrix and $\mathbf{O}_{U \times V}$ denoting an all-zero $U \times V$ matrix. The matrix $\mathbf{E}_{k}$ selects the first $K$ columns of $\mathbf{R}_{x x}$, corresponding to the $K$-channel signal $\overline{\mathbf{x}}_{k}$. The $\mathrm{DANSE}_{K}$ algorithm will compute (10) in an iterative, distributed fashion. Notice that only the first column of $\widehat{\mathbf{W}}_{k}$ is of actual interest, since this is the filter that estimates the desired speech component in the reference microphone. The auxiliary columns of $\widehat{\mathbf{W}}_{k}$ are by-products of the $\mathrm{DANSE}_{K}$ algorithm.

A partitioning of the matrix $\mathbf{W}_{k}$ is defined as $\mathbf{W}_{k}=$ $\left[\mathbf{W}_{k 1}^{T} \cdots \mathbf{W}_{k J}^{T}\right]^{T}$ where $\mathbf{W}_{k q}$ denotes the $M_{k} \times K$ submatrix of $\mathbf{W}_{k}$ that is applied to $\mathbf{y}_{q}$ in (9). Since node $k$ only has access to $\mathbf{y}_{k}$, it can only apply the partial filter $\mathbf{W}_{k k}$. The $K$-channel output signal of this filter, defined by $\mathbf{z}_{k}=\mathbf{W}_{k k}^{H} \mathbf{y}_{k}$, is then broadcast to the other nodes. Another node $q$ can filter this $K$-channel signal $\mathbf{z}_{k}$ that it receives from node $k$ by a MIMO filter defined by the $K \times K$ matrix $\mathbf{G}_{q k}$. This is illustrated in 


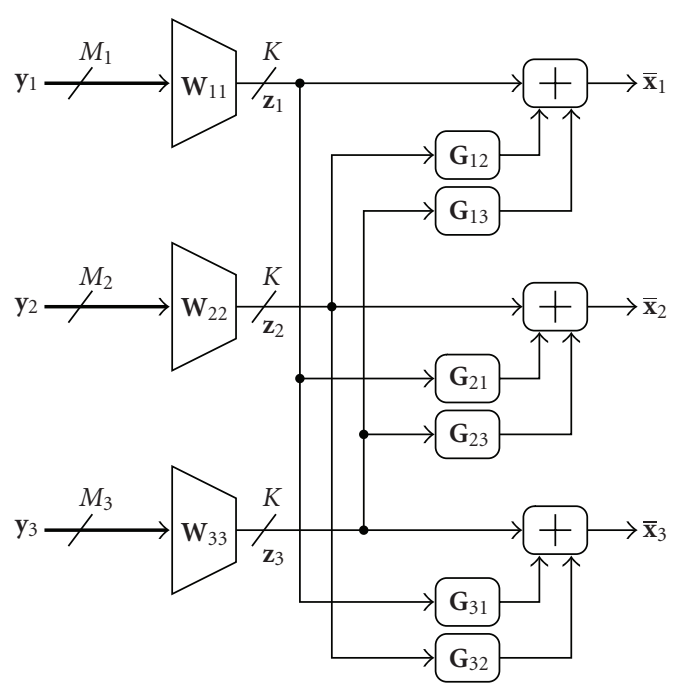

Figure 4: The DANSE $_{K}$ scheme with 3 nodes $(J=3)$. Each node $k$ estimates the desired signal $\overline{\mathbf{x}}_{k}$ using its own $M_{k}$-channel microphone signal, and $2 \mathrm{~K}$-channel signals broadcast by the other two nodes.

Figure 4 for a three-node network $(J=3)$. Notice that the actual $\mathbf{W}_{k}$ that is applied by node $k$ is now parametrized as

$$
\mathbf{W}_{k}=\left[\begin{array}{c}
\mathbf{W}_{11} \mathbf{G}_{k 1} \\
\mathbf{W}_{22} \mathbf{G}_{k 2} \\
\vdots \\
\mathbf{W}_{J J} \mathbf{G}_{k J}
\end{array}\right] .
$$

In what follows, the matrices $\mathbf{G}_{k k}, \forall k \in\{1, \ldots, J\}$, are assumed to be $K \times K$ identity matrices $\mathbf{I}_{K}$ to minimize the degrees of freedom (they are omitted in Figure 4). Node $k$ can only manipulate the parameters $\mathbf{W}_{k k}$ and $\mathbf{G}_{k 1} \cdots \mathbf{G}_{k J}$. If (8) holds, it is shown in [13] that the solution space defined by the parametrization (12) contains the centralized solution $\widehat{\mathbf{W}}_{k}$.

Notice that each node $k$ broadcasts a $K$-channel (Here it is assumed without loss of generality that $K \leq M_{k}, \forall k \in$ $\{1, \ldots, J\}$; if this does not hold at a certain node $k$, this node will transmit its unfiltered microphone signals) signal $\mathbf{z}_{k}$, which is the output of the $M_{k} \times K$ MIMO filter $\mathbf{W}_{k k}$, acting both as a compressor and an estimator at the same time. The subscript $K$ thus refers to the (maximum) number of channels of the broadcast signal. $\mathrm{DANSE}_{K}$ compresses the data to be sent by node $k$ by a factor of $\max \left\{M_{k} / K, 1\right\}$. Further compression is possible, since the channels of the broadcast signal $\mathbf{z}_{k}$ are highly correlated, but this is not taken into consideration throughout this paper.

The $\mathrm{DANSE}_{K}$ algorithm will iteratively update the elements at the righthand side of (12) to optimally estimate the desired signals $\overline{\mathbf{x}}_{k}, \forall k \in\{1, \ldots, J\}$. To describe this updating procedure, the following notation is used.
The matrix $\mathbf{G}_{k}=\left[\mathbf{G}_{k 1}^{T} \cdots \mathbf{G}_{k J}^{T}\right]^{T}$ stacks all transformation matrices of node $k$. The matrix $\mathbf{G}_{k,-q}$ defines the matrix $\mathbf{G}_{k}$ in which $\mathbf{G}_{k q}$ is omitted. The $K(J-1)$-channel signal $\mathbf{z}_{-k}$ is defined as $\mathbf{z}_{-k}=\left[\mathbf{z}_{1}^{T} \cdots \mathbf{z}_{k-1}^{T} \mathbf{z}_{k+1}^{T} \cdots \mathbf{z}_{J}^{T}\right]^{T}$. In what follows, a superscript $i$ refers to the value of the variable at iteration step $i$. Using this notation, the $\mathrm{DANSE}_{K}$ algorithm consists of the following iteration steps:

(1) Initialize

$i \leftarrow 0$

$k \leftarrow 1$

$\forall q \in\{1, \ldots, J\}: \mathbf{W}_{q q} \leftarrow \mathbf{W}_{q q}^{0}, \mathbf{G}_{q,-q} \leftarrow \mathbf{G}_{q,-q}^{0}, \mathbf{G}_{q q} \leftarrow$ $\mathbf{I}_{K}$, where $\mathbf{W}_{q q}^{0}$ and $\mathbf{G}_{q,-q}^{0}$ are random matrices of appropriate dimension.

(2) Node $k$ updates its local parameters $\mathbf{W}_{k k}$ and $\mathbf{G}_{k,-k}$ by solving a local estimation problem based on its own local microphone signals $\mathbf{y}_{k}$ together with the compressed signals $\mathbf{z}_{q}^{i}=\mathbf{W}_{q q}^{i H} \mathbf{y}_{q}$ that it receives from the other nodes $q \neq k$, that is, it minimizes

$$
\widetilde{J}_{k}^{i}\left(\mathbf{W}_{k k}, \mathbf{G}_{k,-k}\right)=E\left\{\left\|\overline{\mathbf{x}}_{k}-\left[\mathbf{W}_{k k}^{H} \mid \mathbf{G}_{k,-k}^{H}\right] \tilde{\mathbf{y}}_{k}^{i}\right\|^{2}\right\},
$$

where

$$
\tilde{\mathbf{y}}_{k}^{i}=\left[\frac{\mathbf{y}_{k}}{\mathbf{z}_{-k}^{i}}\right]
$$

Define $\widetilde{\mathbf{x}}_{k}^{i}$ similarly as (14), but now only containing the desired speech components in the considered signals. The update performed by node $k$ is then

$$
\left[\frac{\mathbf{W}_{k k}^{i+1}}{\mathbf{G}_{k,-k}^{i+1}}\right]=\left(\widetilde{\mathbf{R}}_{y y, k}^{i}\right)^{-1} \widetilde{\mathbf{R}}_{x x, k}^{i} \mathbf{E}_{k}
$$

with

$$
\begin{gathered}
\mathbf{E}_{k}=\left[\begin{array}{c}
\mathbf{I}_{K} \\
\mathbf{O}_{\left(M_{k}-K+K(J-1)\right) \times K}
\end{array}\right], \\
\widetilde{\mathbf{R}}_{y y, k}^{i}=E\left\{\widetilde{\mathbf{y}}_{k}^{i} \widetilde{\mathbf{y}}_{k}^{i H}\right\}, \\
\widetilde{\mathbf{R}}_{x x, k}^{i}=E\left\{\widetilde{\mathbf{x}}_{k}^{i} \widetilde{\mathbf{x}}_{k}^{i H}\right\} .
\end{gathered}
$$

The parameters of the other nodes do not change, that is,

$\forall q \in\{1, \ldots, J\} \backslash\{k\}: \mathbf{W}_{q q}^{i+1}=\mathbf{W}_{q q}^{i}, \mathbf{G}_{q,-q}^{i+1}=\mathbf{G}_{q,-q}^{i}$.

(3) $\mathbf{W}_{k k} \leftarrow \mathbf{W}_{k k}^{i+1}, \mathbf{G}_{k,-k} \leftarrow \mathbf{G}_{k,-k}^{i+1}$

$$
\begin{aligned}
& k \leftarrow(k \bmod J)+1 \\
& i \leftarrow i+1
\end{aligned}
$$

(4) Return to Step 2

Notice that node $k$ updates its parameters $\mathbf{W}_{k k}$ and $\mathbf{G}_{k,-k}$, according to a local multi-channel Wiener filtering problem with respect to its $M_{k}+(J-1) K$ input channels. This MWF 
problem is solved in the same way as the MWF problem given in (3) or (9).

Theorem 1. Assume that $K=$ Q. If $\overline{\mathbf{x}}_{k}=\mathbf{A}_{k} \mathbf{s}, \forall k \in$ $\{1, \ldots, J\}$, with $\mathbf{A}_{k}$ a full rank $K \times K$ matrix, then the DANSE $E_{K}$ algorithm converges for any $k$ to the optimal filters (10) for any initialization of the parameters.

Proof. See [13].

Notice that DANSE $_{K}$ theoretically provides the same output as the centralized MWF algorithm if $K=Q$. The requirement that $\overline{\mathbf{x}}_{k}=\mathbf{A}_{k} \mathbf{s}, \forall k \in\{1, \ldots, J\}$, is satisfied because of (2). However, notice that the data model (2) is only approximately fullfilled in practice due to a finite-length DFT size. Consequently, the rank of the speech correlation matrix $\mathbf{R}_{x x}$ is not $Q$, but it has $Q$ dominant eigenvalues instead. Therefore, the theoretical claims of convergence and optimality of $\operatorname{DANSE}_{K}$, with $K=Q$, are only approximately true in practice due to frequency domain processing.

4.2. Simultaneous Updating. The $\mathrm{DANSE}_{K}$ algorithm as described in Section 4.1 performs sequential updating in a round-robin fashion, that is, nodes update their parameters one at a time. In [20], it is observed that convergence of DANSE is no longer guaranteed when nodes update simultaneously, or in an uncoordinated fashion where each node decides independently in which iteration steps it updates its parameters. This is however an interesting case, since a simultaneous updating procedure allows for parallel computation, and uncoordinated updating removes the need for a network wide protocol that coordinates the updates between nodes.

Let $\mathbf{W}=\left[\mathbf{W}_{11}^{T} \mathbf{W}_{22}^{T} \cdots \mathbf{W}_{J J}^{T}\right]^{T}$, and let $F(\mathbf{W})$ be the function that defines the simultaneous DANSE $_{K}$ update of all parameters in $\mathbf{W}$, that is, $F$ applies (15) $\forall k \in\{1, \ldots J\}$ simultaneously. Experiments in [20] show that the update $\mathbf{W}^{i+1}=F\left(\mathbf{W}^{i}\right)$ may lead to limit cycle behavior. To avoid these limit cycles, the following relaxed version of DANSE is suggested in [20]:

$$
\mathbf{W}^{i+1}=\left(1-\alpha^{i}\right) \mathbf{W}^{i}+\alpha^{i} F\left(\mathbf{W}^{i}\right)
$$

with stepsizes $\alpha^{i}$ satisfying

$$
\begin{aligned}
& \alpha^{i} \in(0,1], \\
& \lim _{i \rightarrow \infty} \alpha^{i}=0, \\
& \sum_{i=0}^{\infty} \alpha^{i}=\infty .
\end{aligned}
$$

The suggested conditions on the stepsize $\alpha^{i}$ are however quite conservative and may result in slow convergence. In most cases, the simultaneous update procedure converges already when a constant value for $\alpha^{i}$ is chosen $\forall i \in \mathbb{N}$ that is sufficiently small. In all simulations performed for the scenario in Section 3, a value of $\alpha^{i}=0.5, \forall i \in \mathbb{N}$ was found to eliminate limit cycles in every setup.

\section{Robust DANSE}

5.1. Robustness Issues in DANSE. In Section 6, simulation results will show that the DANSE algorithm does not achieve the optimal noise reduction performance as predicted by Theorem 1. There are two important reasons for this suboptimal performance.

The first reason is the fact that the $\mathrm{DANSE}_{K}$ algorithm assumes that the signal space spanned by the channels of $\overline{\mathbf{x}}_{k}$ is well-conditioned, $\forall k \in\{1, \ldots, J\}$. This assumption is reflected in Theorem 1 by the condition that $\mathbf{A}_{k}$ be full rank for all $k$. Although this is mostly satisfied in practice, the $\mathbf{A}_{k}$ 's are often ill-conditioned. For instance, the distance between microphones in a single node is mostly small, yielding a steering matrix with several columns that are almost identical, that is, an ill-conditioned matrix $\mathbf{A}_{k}$ in the formulation of Theorem 1 .

The microphones of nodes that are close to a noise source typically collect low SNR signals. Despite the low SNR, these signals can boost the performance of the MWF algorithm, since they can act as noise references to cancel out noise in the signals recorded by other nodes. However, the DANSE algorithm cannot fully exploit this since the local estimation problem at such low SNR nodes is illconditioned. If node $k$ has low SNR microphone signals $\mathbf{y}_{k}$, the correlation matrix $\overline{\mathbf{R}}_{x x, k}=E\left\{\overline{\mathbf{x}}_{k} \overline{\mathbf{x}}_{k}^{H}\right\}$ has large estimation errors, since the corresponding noise correlation matrix $\overline{\mathbf{R}}_{v v, k}$ and the speech+noise correlation matrix $\overline{\mathbf{R}}_{y y, k}$ are very similar, that is, $\overline{\mathbf{R}}_{v v, k} \approx \overline{\mathbf{R}}_{y y, k}$. Notice that $\overline{\mathbf{R}}_{x x, k}$ is a submatrix of $\widetilde{\mathbf{R}}_{x x, k}$ defined in (18), which is used in the $\operatorname{DANSE}_{K}$ algorithm. From another point of view, this also relates to an ill-conditioned steering matrix $\mathbf{A}$, since the submatrix $\mathbf{A}_{k}$ is close to an all-zero matrix compared to the submatrices corresponding to nodes with higher SNR signals.

5.2. Robust DANSE (R-DANSE). In this section, a modification to the DANSE algorithm is proposed to achieve a better noise reduction performance in the case of low SNR nodes or ill-conditioned steering matrices. The main idea is to replace an ill-conditioned $\mathbf{A}_{k}$ matrix by a better conditioned matrix by changing the estimation problem at node $k$. The new algorithm is referred to as "robust DANSE" or R-DANSE. In what follows, the notation $v(p)$ is used to denote the $p$ th entry in a vector $\mathbf{v}$, and $\mathbf{m}(p)$ is used to denote the $p$-th column in the matrix $\mathbf{M}$.

For each node $k$, the channels in $\overline{\mathbf{x}}_{k}$ that cause illconditioned steering matrices, or that correspond to low SNR signals, are discarded and replaced by the desired speech components in the signal(s) $\mathbf{z}_{q}^{i}$ received from other (high SNR) nodes $q \neq k$, that is,

$$
\bar{x}_{k}^{i}(p)=\mathbf{w}_{q q}^{i}(l)^{H} \mathbf{x}_{q}, q \in\{1, \ldots, J\} \backslash\{k\}, l \in\{1, \ldots, K\},
$$

if $x_{k p}$ causes an ill-conditioned steering matrix or if $x_{k p}$ corresponds to a low SNR microphone, and

$$
\bar{x}_{k}^{i}(p)=x_{k p}
$$


otherwise. Notice that the desired signal $\overline{\mathbf{x}}_{k}^{i}$ may now change at every iteration, which is reflected by the superscript $i$ denoting the iteration index.

To decide whether to use (24) or (25), the condition number of the matrix $\mathbf{A}_{k}$ does not necessarily have to be known. In principle, it is always better to replace the $K-1$ auxiliary channels in $\overline{\mathbf{x}}_{k}$ as in formula (24), where a different $q$ should be chosen for every $p$. Indeed, since microphones of different nodes are typically far apart from each other, better conditioned steering matrices are then obtained. Also, since the correlation matrix $\widetilde{\mathbf{R}}_{x x, k}$ is better estimated when high SNR signals are available, the chosen $q$ 's preferably correspond to high SNR nodes. Therefore, the decision procedure requires knowledge of the SNR at the different nodes. For a low SNR node $k$, one can also replace all $K$ channels in $\overline{\mathbf{x}}_{k}$ as in (24), including the reference microphone. In this case, there is no estimation of the speech component that is collected by the microphones of node $k$ itself. However, since the network wide problem is now better conditioned, the other nodes in the network will benefit from this.

The R-DANSE $E_{K}$ algorithm performs the same steps as explained in Section 4.1 for the DANSE $_{K}$ algorithm, but now $\overline{\mathbf{x}}_{k}^{i}$ replaces $\overline{\mathbf{x}}_{k}$ in (13)-(18). This means that in R-DANSE, the $\mathbf{E}_{k}$ matrix in (16) now may contain ones at row indices that are higher than $M_{k}$. To guarantee convergence of R-DANSE, the placement of ones in (16), or equivalently the choices for $q$ and $l$ in (24), is not completely free, as explained in the next section.

5.3. Convergence of R-DANSE. To provide convergence results, the dependencies of each individual estimation problem are described by means of a directed graph $g$ with $K J$ vertices, where each vertex corresponds to one of the locally computed filters, that is, a specific column of $\mathbf{W}_{k k}$ for $k=1 \cdots J$. (Readers that are not familiar with the jargon of graph theory might want to consult [23], although in principle no prior knowledge on graph theory is assumed). The graph contains an arc from filter $a$ to $b$, described by the ordered pair $(a, b)$, if the output of filter $b$ contains the desired speech component that is estimated by filter $a$. For example, formula (24) defines the $\operatorname{arc}\left(\mathbf{w}_{k k}(p), \mathbf{w}_{q q}(l)\right)$. A vertex $v$ that has no departing arc is referred to as a direct estimation filter (DEF), that is, the signal to be estimated is the desired speech component in one of the node's own microphone signals, as in formula (25).

To illustrate this, a possible graph is shown in Figure 5 for DANSE 2 applied to the scenario described in Section 3, where the hearing aid users are now listening to two speakers, that is, speakers B and C. Since the microphone signals of node 1 have a low SNR, the two desired signals in $\overline{\mathbf{x}}_{1}$ that are used in the computation of $\mathbf{W}_{11}$ are replaced by the filtered desired speech component in the received signals from higher SNR nodes 2 and 4, that is, $\mathbf{w}_{22}(1)^{H} \mathbf{x}_{2}$ and $\mathbf{w}_{44}(1)^{H} \mathbf{x}_{4}$, respectively. This corresponds to the $\operatorname{arcs}\left(\mathbf{w}_{11}(1), \mathbf{w}_{22}(1)\right)$ and $\left(\mathbf{w}_{11}(2), \mathbf{w}_{44}(1)\right)$. To calculate $\mathbf{w}_{22}(1), \mathbf{w}_{33}(1)$, and $\mathbf{w}_{44}(1)$, the desired speech components $x_{21}, x_{31}$ and $x_{41}$ in the respective reference microphones are used. These filters

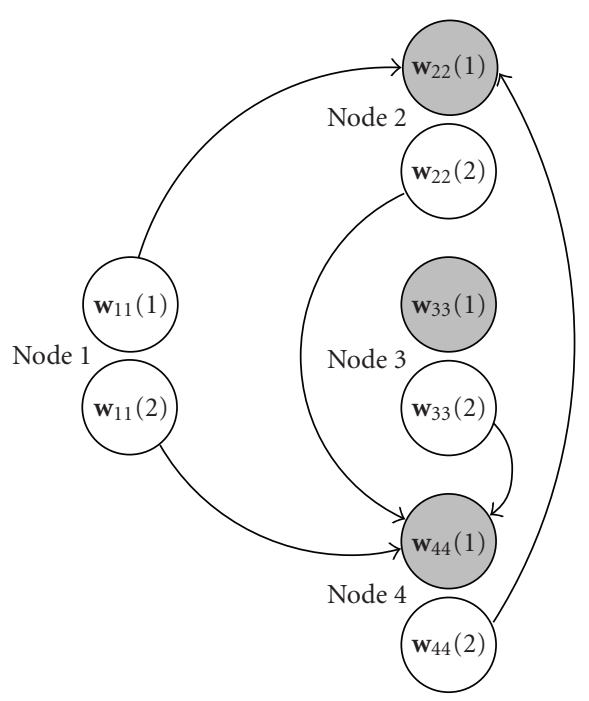

Figure 5: Possible graph describing dependencies of estimations problems for DANSE 2 applied to the acoustic scenario described in Section 3.

are DEF's, and are shaded in Figure 5. The microphones at node 2 are very close to each other. Therefore, to avoid an illconditioned matrix $\mathbf{A}_{2}$ at node 2, the signals to be estimated by $\mathbf{w}_{22}(2)$ should be provided by another node, and not by another microphone signal of node 2 itself. Therefore, the $\operatorname{arc}\left(\mathbf{w}_{22}(2), \mathbf{w}_{44}(1)\right)$ is added. For similar reasons, the arcs $\left(\mathbf{w}_{33}(2), \mathbf{w}_{44}(1)\right)$ and $\left(\mathbf{w}_{44}(2), \mathbf{w}_{22}(1)\right)$ are also added.

Theorem 2. Let all assumptions of Theorem 1 be satisfied. Let $g$ be the directed graph describing the dependencies of the estimation problems in the R-DANSE $E_{K}$ algorithm as described above. If $g$ is acyclic, then the R-DANSE $E_{K}$ algorithm converges to the optimal filters to estimate the desired signals defined byg.

Proof. The proof of Theorem 1 in [13] on convergence of DANSE $_{K}$ is based on the assumption that the desired $K$ channel signals $\overline{\mathbf{x}}_{k}, \forall k \in\{1, \ldots, J\}$, are all in the same $K$ dimensional signal subspace spanned by the $K$ sources in $\mathbf{s}$, that is,

$$
\overline{\mathbf{x}}_{k}=\mathbf{A}_{k} \mathbf{s}
$$

This assumption remains valid in R-DANSE $E_{K}$. Indeed, since $\mathbf{x}_{q}$ contains $M_{q}$ linear combination of the $Q$ sources in $\mathbf{s}$, the signal $\bar{x}_{k}^{i}(p)$ given by (24) is again a linear combination of the source signals. However, the coefficients of this linear combinations may change at every iteration as the signal $\bar{x}_{k}^{i}(p)$ is an output of the adaptive filter $\mathbf{w}_{q q}^{i}(l)$ in another node $q$. This then leads to a modified version of Theorem 1 for DANSE $E_{K}$ in which the matrix $\mathbf{A}_{k}$ in (26) is not fixed, but may change at every iteration, that is,

$$
\overline{\mathbf{x}}_{k}^{i}=\mathbf{A}_{k}^{i} \mathbf{s}
$$


Define

$$
\overline{\mathbf{W}}_{k q}^{i}=\underset{\mathbf{W}_{k q}}{\arg \min }\left(\min _{\mathbf{G}_{k,-q}} E\left\{\left\|\overline{\mathbf{x}}_{k}-\left[\mathbf{W}_{k q}^{H} \mid \mathbf{G}_{k,-q}^{H}\right] \tilde{\mathbf{y}}_{q}^{i}\right\|^{2}\right\}\right) \text {. }
$$

This corresponds to the hypothetical case in which node $k$ would optimise $\mathbf{W}_{k q}^{i}$ directly, without the constraint $\mathbf{W}_{k q}^{i}=$ $\mathbf{W}_{q q}^{i} \mathbf{G}_{k q}^{i}$ where node $k$ depends on the parameter choice of node $q$.

In [13] it is proven that for $\operatorname{DANSE}_{K}$, under the assumptions of Theorem 1, the following holds:

$$
\forall q, k \in\{1, \ldots, J\}: \overline{\mathbf{W}}_{k q}^{i}=\overline{\mathbf{W}}_{q q}^{i} \mathbf{A}_{k q}
$$

with $\mathbf{A}_{k q}=\mathbf{A}_{q}^{-H} \mathbf{A}_{k}^{H}$. This means that the columns of $\overline{\mathbf{W}}_{q q}^{i}$ span a $K$-dimensional subspace that also contains the columns of $\overline{\mathbf{W}}_{k q}^{i}$, which is the optimal update with respect to the cost function $J_{k}^{i}$ of node $k$, as if there were no constraints on $\mathbf{W}_{k q}^{i}$. Or in other words, an update by node $q$ automatically optimizes the cost function of any other node $k$ with respect to $\mathbf{W}_{k q}$, if node $k$ performs a responding optimization of $\mathbf{G}_{k q}$, yielding $\mathbf{G}_{k q}^{\text {opt }}=\mathbf{A}_{k q}$. Therefore, the following expression holds:

$$
\begin{aligned}
\forall k \in\{1, \ldots, J\}, \forall i \in \mathbb{N}: & \min _{\mathbf{G}_{k,-k}} \widetilde{J}_{k}^{i+1}\left(\mathbf{W}_{k k}^{i+1}, \mathbf{G}_{k,-k}\right) \\
& \leq \min _{\mathbf{G}_{k,-k}} \widetilde{J}_{k}^{i}\left(\mathbf{W}_{k k}^{i}, \mathbf{G}_{k,-k}\right) .
\end{aligned}
$$

Notice that this holds at every iteration for every node. In the case of R-DANSE ${ }_{K}$, the $\mathbf{A}_{k q}$ matrix of expression (29) changes at every iteration. At first sight, expression (30) remains valid, since changes in the matrix $\mathbf{A}_{k q}$ are compensated by the minimization over $\mathbf{G}_{k q}$ in (30). However, this is not true since the desired signals $\overline{\mathbf{x}}_{k}^{i}$ also change at every iteration, and therefore the cost functions at different iterations cannot be compared.

Expression (30) can be partitioned in $K$ sub-expressions:

$$
\begin{gathered}
\forall p \in\{1, \ldots, K\}, \forall k \in\{1, \ldots, J\}, \quad \forall i \in \mathbb{N}: \\
\min _{\mathbf{g}_{k,-k}(p)} \widetilde{J}_{k p}^{i+1}\left(\mathbf{w}_{k k}^{i+1}(p), \mathbf{g}_{k,-k}(p)\right) \leq \min _{\mathbf{g}_{k,-k}(p)} \widetilde{\widetilde{l}}_{k p}^{i}\left(\mathbf{w}_{k k}^{i}(p), \mathbf{g}_{k,-k}(p)\right)
\end{gathered}
$$

with

$$
\tilde{J}_{k p}^{i}\left(\mathbf{w}_{k k}, \mathbf{g}_{k,-k}\right)=E\left\{\left|\bar{x}_{k}(p)-\left[\mathbf{w}_{k k}^{H} \mid \mathbf{g}_{k,-k}^{H}\right] \tilde{\mathbf{y}}_{k}^{i}\right|^{2}\right\} .
$$

For the R-DANSE ${ }_{K}$ case, (33) remains the same, except that $\bar{x}_{k}(p)$ has to be replaced with $\bar{x}_{k}^{i}(p)$. As explained above, due to this modification, expression (32) does not hold anymore. However, it does hold for the cost functions $J_{k p}^{i}$ corresponding to a DEF $\mathbf{w}_{k k}(p)$, that is, a filter for which the desired signal is directly obtained from one of the microphone signals of node $k$. Indeed, every DEF $\mathbf{w}_{k k}(p)$ has a well-defined cost function $\widetilde{J}_{k p}^{i}$, since the signal $\bar{x}_{k}^{i}(p)$ is fixed over different iteration steps. Because $\widetilde{J}_{k p}^{i}$ has a lower bound, (32) shows that the sequence $\left\{\min _{\mathbf{g}_{k, k}^{p}} \tilde{J}_{k p}^{i}\right\}_{i \in \mathbb{N}}$ converges. The convergence of this sequence implies convergence of the sequence $\left\{\mathbf{w}_{k k}^{i}(p)\right\}_{i \in \mathbb{N}}$, as shown in [13].

After convergence of all $\mathbf{w}_{k k}(p)$ parameters corresponding to a DEF, all vertices in the graph $g$ that are directly connected to this DEF have a stable desired signal, and their corresponding cost functions become well-defined. The above argument shows that these filters then also converge.

Continuing this line of thought, convergence properties of the DEF will diffuse through the graph. Since the graph is acyclic, all vertices converge. Convergence of all $\mathbf{W}_{k k}$ parameters for $k=1 \cdots J$ automatically yields convergence of all $\mathbf{G}_{k}$ parameters, and therefore convergence of all $\mathbf{W}_{k}$ filters for $k=1 \cdots J$. Optimality of the resulting filters can be proven using the same arguments as in the optimality proof of Theorem 1 for DANSE $_{K}$ in [13].

\section{Performance of DANSE and R-DANSE}

In this section, the batch mode performance of DANSE and R-DANSE is compared for the acoustic scenario of Section 3. In this batch version of the algorithms, all iterations of DANSE and R-DANSE are on the full signal length of about 20 seconds. In real-life applications, however, iterations will of course be spread over time, that is, subsequent iterations are performed on different signal segments. To isolate the influence of VAD errors, an ideal VAD is used in all experiments. Correlation matrices are estimated by time averaging over the complete length of the signal. The sampling frequency is $32 \mathrm{kHz}$ and the DFT size is equal to $L=512$ if not specified otherwise.

6.1. Experimental Validation of DANSE and R-DANSE. Three different measures are used to assess the quality of the outputs at the hearing aids: the signal-to-noise ratio (6), the signal-to-distortion ratio (7), and the mean squared error (MSE) between the coefficients of the centralized multichannel Wiener filter $\hat{\mathbf{w}}_{k}$ and the filter obtained by the DANSE algorithm, that is,

$$
\operatorname{MSE}=\frac{1}{L} \sum\left\|\widehat{\mathbf{w}}_{k}-\mathbf{w}_{k}(1)\right\|^{2}
$$

where the summation is performed over all DFT bins, with $L$ the DFT size, $\hat{\mathbf{w}}_{k}$ defined by (4), and $\mathbf{w}_{k}(1)$ denoting the first column of $\mathbf{W}_{k}$ in (12), that is, the filter that estimates the speech component $x_{k 1}$ in the reference microphone at node $k$.

Two different scenarios are tested. In scenario 1 the dimension $Q$ of the desired signal space is $Q=1$, that is, both hearing aid users are listening to speaker $C$, whereas speakers $\mathrm{A}$ and $\mathrm{B}$ and the babble-noise loudspeaker are considered to be background noise. In Figure 6, the three quality measures are plotted (for node 4 ) versus the iteration index for DANSE 1 and R-DANSE ${ }_{1}$, with either sequential updating or simultaneous updating (without relaxation). Also an upper bound is plotted, which corresponds to the centralized MWF solution defined in (4). The R-DANSE 1 


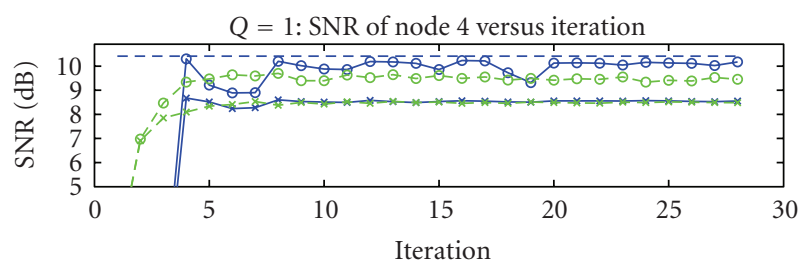

(a)

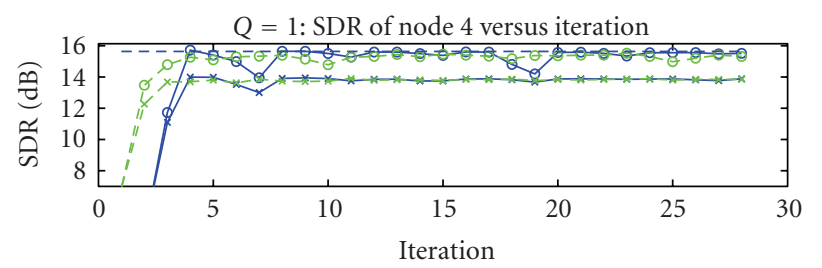

(b)

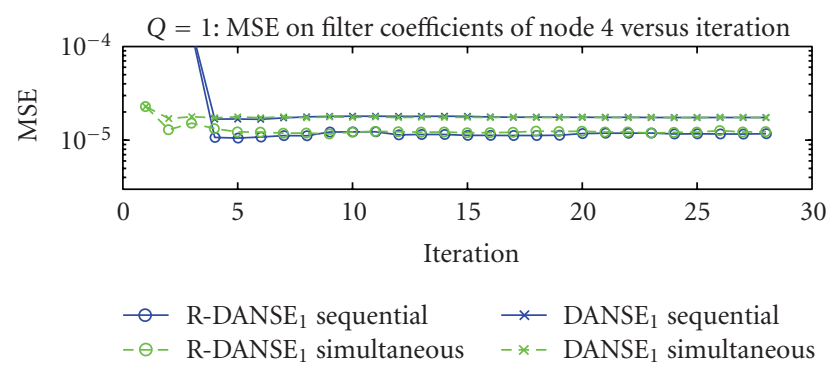

(c)

FIgURE 6: Scenario 1: SNR, SDR, and MSE on filter coefficients versus iterations for DANSE $_{1}$ and R-DANSE 1 at node 4 , for both sequential and simultaneous updates. Speaker $\mathrm{C}$ is the only target speaker.

graph consists of only DEF nodes, except for $\mathbf{w}_{11}$, which has an $\operatorname{arc}\left(\mathbf{w}_{11}, \mathbf{w}_{44}\right)$ to avoid performance loss due to low SNR. Since there is only one desired source, DANSE $_{1}$ theoretically should converge to the upper bound performance, but this is

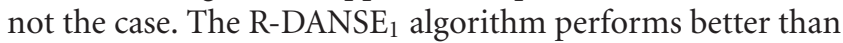
the DANSE $_{1}$ algorithm, yielding an SNR increase of 1.5 to $2 \mathrm{~dB}$, which is an increase of about $20 \%$ to $25 \%$. The same holds for the other two hearing aids, that is, node 2 and 3 , which are not shown here. The parallel update typically converges faster but it converges to a suboptimal limit cycle, since no relaxation is used. Although this limit cycle is not very clear in these plots, a loss in SNR of roughly $1 \mathrm{~dB}$ is observed in every hearing aid. This can be avoided by using relaxation, which will be illustrated in Section 6.2.

In scenario 2 , the case in which $Q=2$ is considered, that is, there are two desired sources: both hearing aid users are listening to speakers B and C, who talk simultaneously, yielding a speech correlation matrix $\mathbf{R}_{x x}$ of approximately rank 2. The R-DANSE ${ }_{2}$ graph is illustrated in Figure 5. For this 2-speaker case, both $\mathrm{DANSE}_{1}$ and $\mathrm{DANSE}_{2}$ are evaluated, where the latter should theoretically converge to the upper bound performance. The results for node 4 are plotted in Figure 7. While the MSE is lower for $\mathrm{DANSE}_{2}$ compared to $\mathrm{DANSE}_{1}$, it is observed that $\mathrm{DANSE}_{2}$ does not reach the optimal noise reduction performance. R-DANSE 2

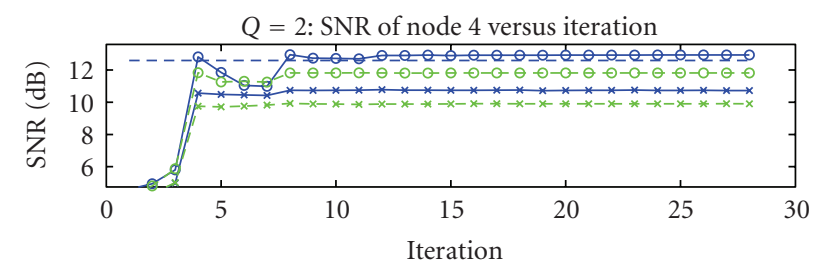

(a)

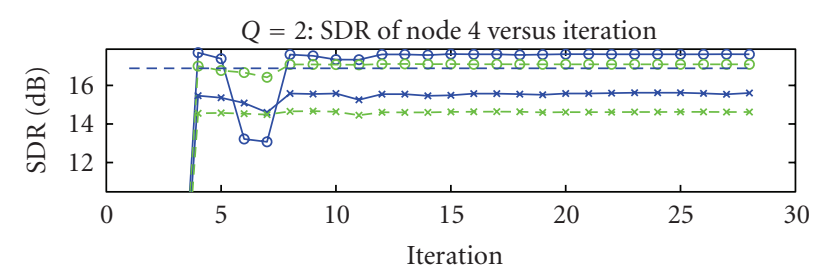

(b)

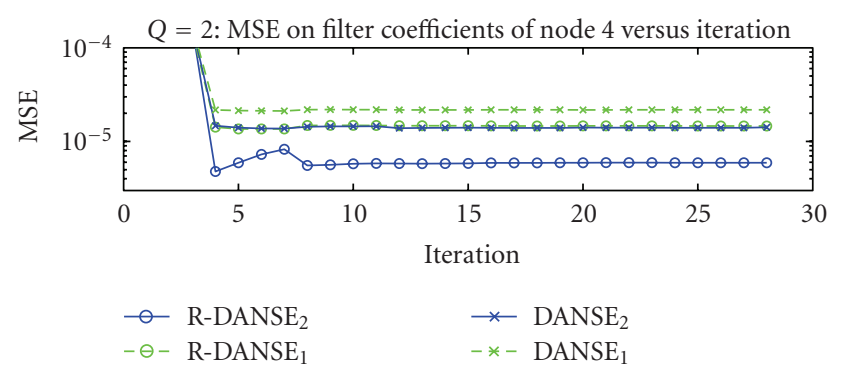

(c)

Figure 7: Scenario 2: SNR, SDR and MSE on filter coefficients versus iterations for DANSE 1 , R-DANSE 1, DANSE $_{2}$ and R-DANSE 2 at node 4 . Speakers $B$ and $C$ are target speakers.

is however able to reach the upper bound performance at every hearing aid. The SNR improvement of R-DANSE 2 in comparison with $\mathrm{DANSE}_{2}$ is between 2 and $3 \mathrm{~dB}$ at every hearing aid, which is again an increase of about $20 \%$ to $25 \%$. Notice that R-DANSE 2 even slightly outperforms the centralized algorithm. This may be because R-DANSE 2 performs its matrix inversions on correlation matrices with smaller dimensions than the all-microphone correlation matrix $\mathbf{R}_{y y}$ in the centralized algorithm, which is more favorable in a numerical sense.

6.2. Simultaneous Updating with Relaxation. Simulations on different acoustic scenarios show that in most cases, DANSE $_{K}$ with simultaneous updating results in a limit cycle oscillation. The occurrence of limit cycles appears to depend on the position of the nodes and sound sources, the reverberation time, as well as on the DFT size, but no clear rule was found to predict the occurrence of a limit cycle.

To illustrate the effect of relaxation, the simulation results of R-DANSE 1 in the scenario of Section 3 are given in Figure $8(\mathrm{a})$, where now the DFT size is $L=1024$, which results in clearly visible limit cycle oscillations when no relaxation is used. This causes an over-all loss in SNR of 2 or $3 \mathrm{~dB}$ at every hearing aid.

Figure 8 (b) shows the same experiment where relaxation is used as in formula (20) with $\alpha^{i}=0.5, \forall i \in \mathbb{N}$. 

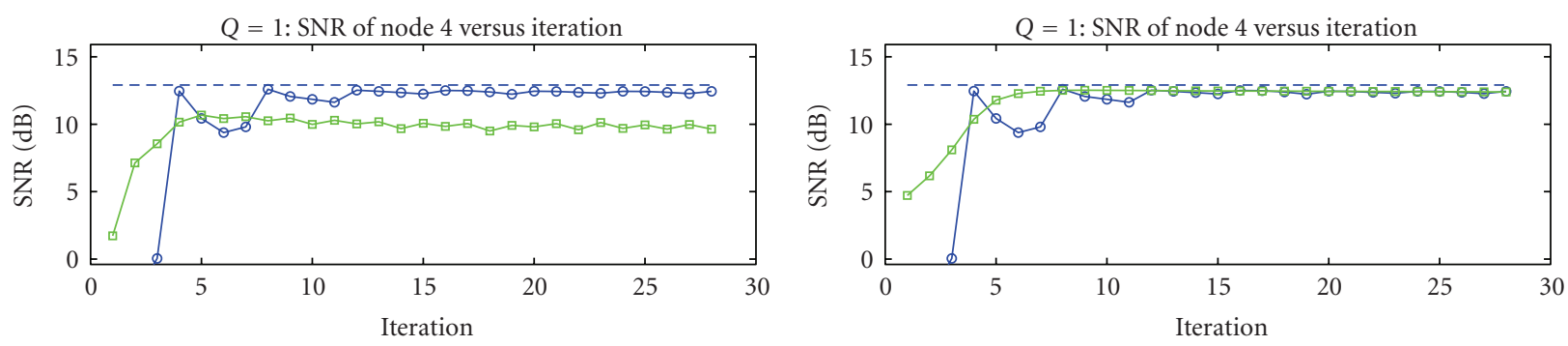

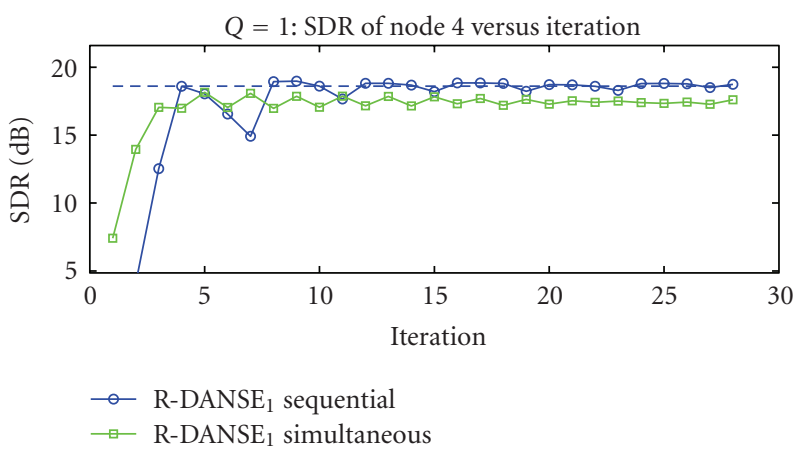

(a) without relaxation

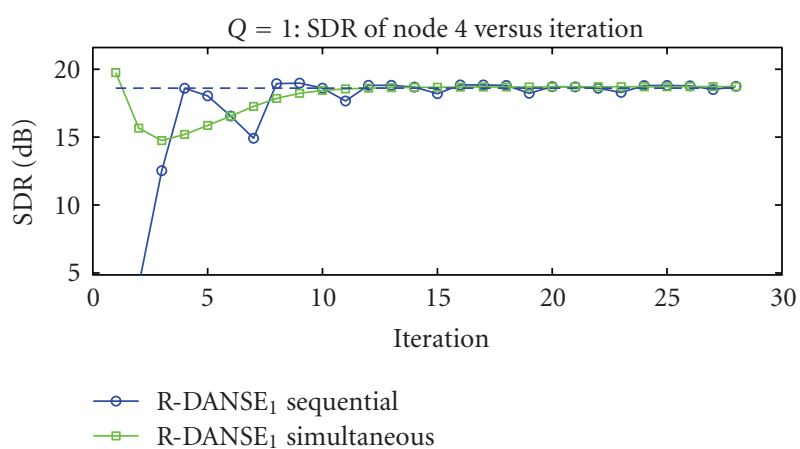

(b) with relaxation $\left(\alpha^{i}=0.5, \forall i \in \mathbb{N}\right)$

FIGURE 8: SNR and SDR for R-DANSE ${ }_{1}$ versus iterations at node 4 with sequential and simultaneous updating.

In this case, the limit cycle does not appear and the simultaneous updating algorithm indeed converges to the same values as the sequential updating algorithm. Notice that the simultaneous updating algorithm converges faster than the sequential updating algorithm.

6.3. DFT Size. In Figure 9, the SNR and SDR of the output signal of R-DANSE ${ }_{1}$ at nodes 3 and 4 is plotted as a function of the DFT size $L$, which is equivalent to the length of the time domain filters that are implicitly applied to the signals at the nodes. 28 iterations were performed with sequential updating for $L=256, L=512, L=1024$, and $L=2048$. The outputs of the centralized version and the scenario in which nodes do not share any signals, are also given as a reference.

As expected, the performance increases with increasing DFT size. However, the discrepancy between the centralized algorithm and R-DANSE 1 grows for increasing DFT size. One reason for this observation is that, for large DFT sizes, R-DANSE often converges slowly once the filters at all nodes are close to the optimal filters.

The scenario with isolated nodes is less sensitive to the DFT size. This is because the tested DFT sizes are quite large, yielding long filters. As explained in the next section, shorter filter lengths are sufficient in the case of isolated nodes since the microphones are very close to each other, yielding small time differences of arrival (TDOA).

6.4. Communication Delays or Time Differences of Arrival. To exploit the spatial coherence between microphone signals, the noise reduction filters attempt to align the signal components resulting from the same source in the different microphone signals. However, alignment of the direct components of the source signals is only possible when the filter lengths are at least twice the maximum time difference of arrival (TDOA) between all the microphones. This means that in general, the noise reduction performance degrades with increasing TDOA's and fixed filter lengths. Large TDOA's require longer filters, or appropriate delay compensation. As already mentioned in Section 3, delay compensation is restricted in hearing aids due to lip synchronization constraints.

The TDOA depends on the distance between the microphones, the position of the sources and the delay introduced by the communication link. Figure 10 shows the performance degradation of R-DANSE at nodes 3 and 4 when the TDOA increases, in this case modelled by an increasing communication delay between the nodes. There is no delay compensation, that is, none of the signals are delayed before filtering. DFT sizes $L=512$ and $L=1024$ are evaluated. The outputs of the centralized MWF procedure are also given as a reference, as well as the procedure where every node broadcasts its first microphone signal, which corresponds to the scenario in which all supporting nodes are single-microphone nodes. The lower bound is defined by the scenario where all nodes are isolated, that is, each node only uses its own microphones in the estimation process.

As expected, when the communication delay increases, the performance degrades due to increasing time lags between signals. At node 3, the R-DANSE algorithm is slightly more sensitive to the communication delay than the centralized MWF. The behavior at node 2 is very similar, and is omitted here. Furthermore, for large communication delays, R-DANSE is outperformed by the single-microphone nodes scenario. At node 4, both the centralized MWF and 

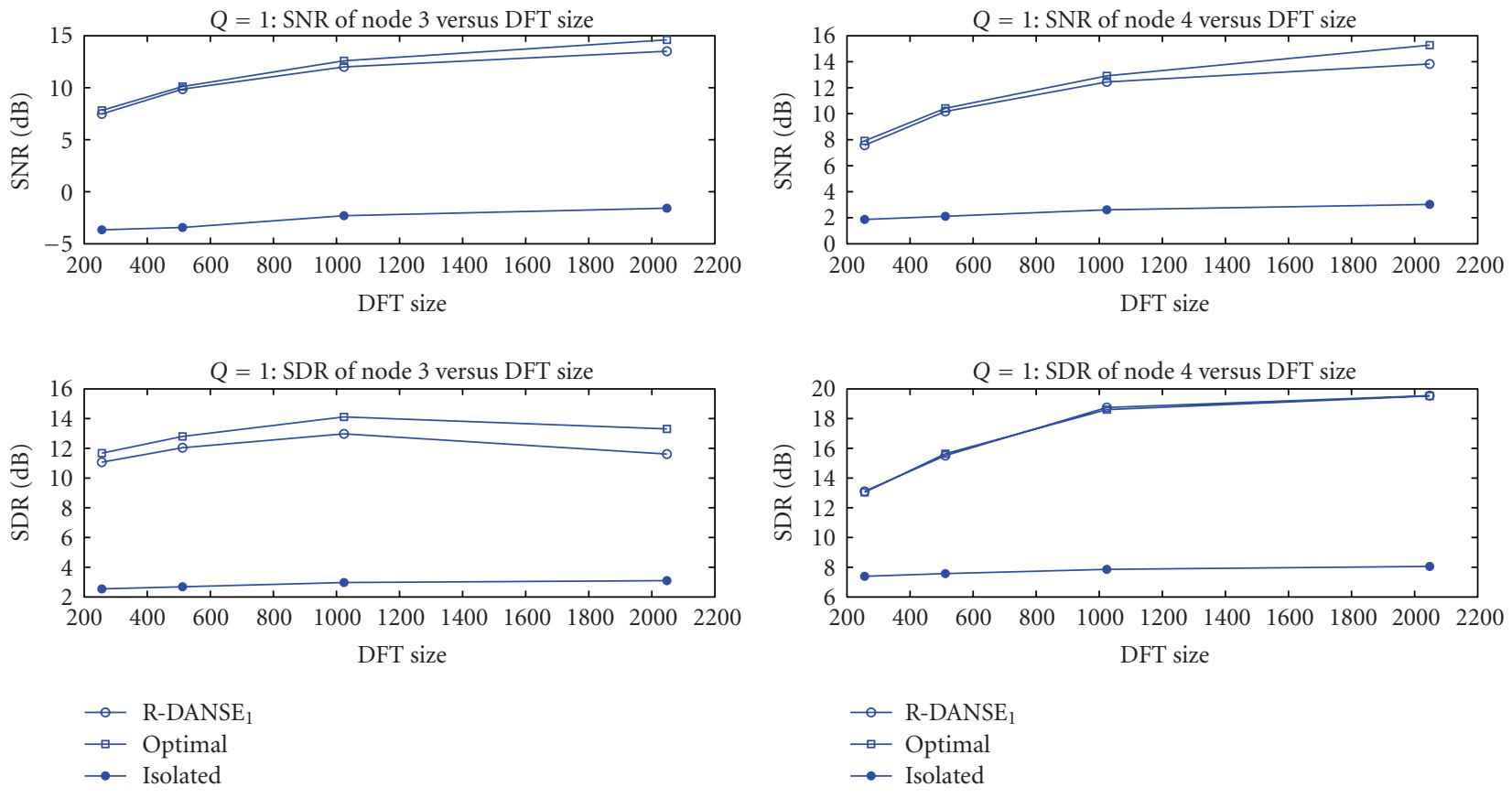

(b) node 4

FIGURE 9: Output SNR and SDR after 28 iterations of R-DANSE w $_{1}$ ith sequential updating versus DFT size $L$ at nodes 3 and 4.
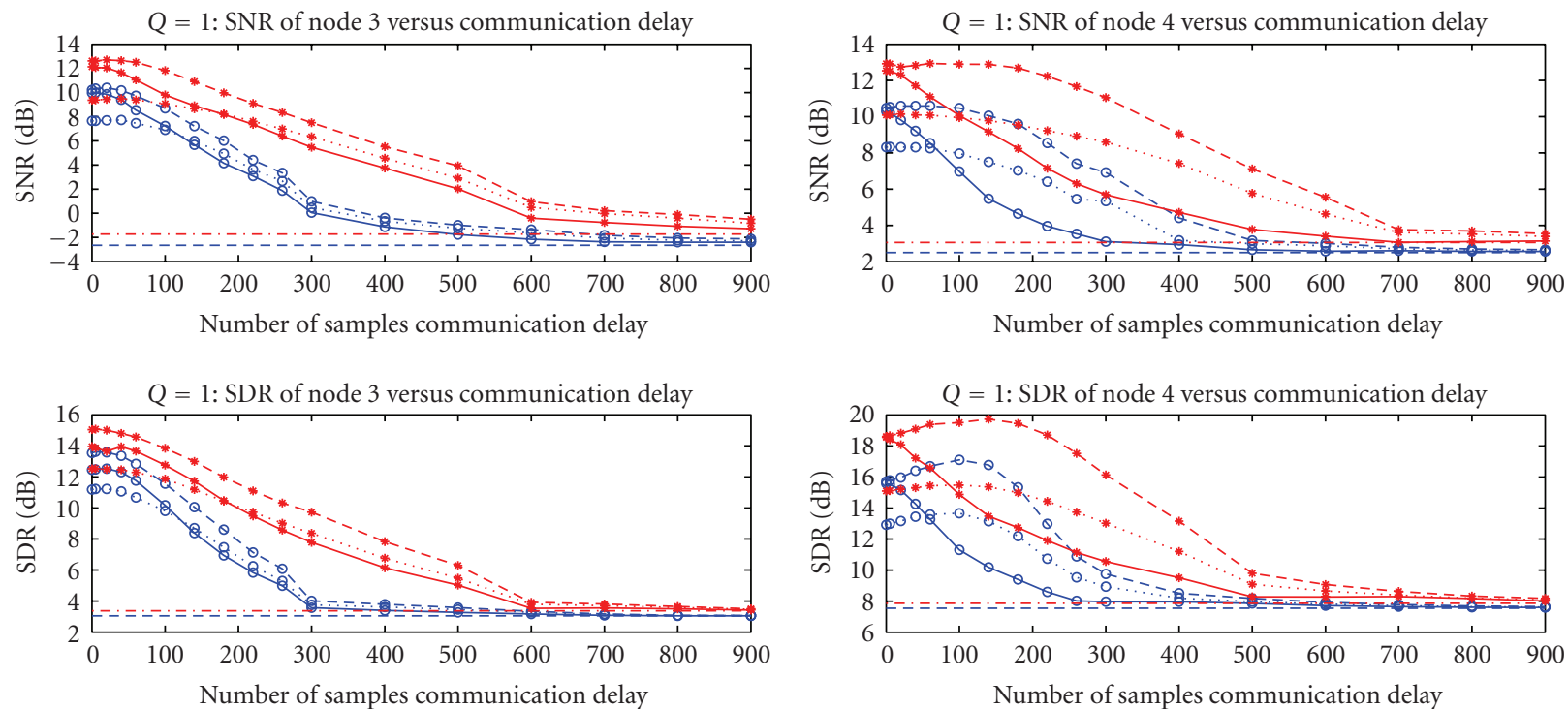

\footnotetext{
$\rightarrow$ R-DANSE $L=512$

$-\theta$ - Centralized $L=512$

. o. One mic of other nodes $L=512$

- - - Isolated $L=512$

* * R-DANSE $L=1024$

- * - Centralized $L=1024$

**. One mic of other nodes $L=1024$

- . Isolated $L=1024$
}

(a) node 3

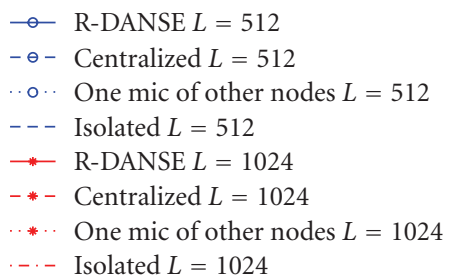

(b) node 4

FIGURE 10: Output SNR and SDR at nodes 3 and 4 after 12 iterations of R-DANSE 1 with sequential updating vs. delay of the communication link. 
the single-microphone nodes scenario even benefit from communication delays. Apparently, the additional delay allows the estimation process to align the signals more effectively.

The reason why R-DANSE is more sensitive to a communication delay than the centralized MWF is that the latter involves independent estimation processes, whereas in RDANSE, the estimation at any node $k$ depends on the quality of estimation at every other node $q \neq k$. Notice however that the influence of communication delay is of course very dependent on the scenario and its resulting TDOA's. The above results only give an indication of this influence.

\section{Practical Issues and Open Problems}

In the batch-mode simulations provided in this paper, some practical aspects have been disregarded. Therefore, the actual performance of the MWF and the $\mathrm{DANSE}_{K}$ algorithm may be worse than what is shown in the simulations. In this section, some of these practical aspects are briefly discussed.

The VAD is a crucial ingredient in MWF-based noise reduction applications. A simple VAD may not behave well in the simulated scenario as described in Figure 2 due to the fact that the noise component also contains competing speech signals. Especially the VADs at nodes that are close to an interfering speech source (e.g., node 1 in Figure 2) are bound to make many wrong decisions, which will then severely deteriorate the output of the DANSE algorithm. To solve this, a speaker selective VAD should be used, for example, [24]. Also, low SNR nodes should be able to use VAD information from high SNR nodes. By sharing VAD information, better VAD decisions can be made [25]. How to organize this, and how a consensus decision can be found between different nodes, is still an open research problem.

A related problem is the actual selection of the desired source, versus the noise sources. A possible strategy is that the speech source with the highest power at a certain reference node is selected as the desired source. In hearing aid applications, it is often assumed that the desired source is in front of the listener. Since the actual positions of the hearing aid microphones are known (to a certain accuracy), the VAD can be combined with a source localization algorithm or a fixed beamformer to distinguish between a target speaker and an interfering speaker. Again, this information should be shared between nodes so that all nodes can eventually make consistent selections.

A practical aspect that needs special attention is the adaptive estimation of the correlation matrices in the DANSE $_{K}$ algorithm. In many MWF implementations, correlation matrices are updated with the instantaneous sample correlation matrix and by using a forgetting factor $0<\lambda<1$, that is,

$$
\mathbf{R}_{y y}[t]=\lambda \mathbf{R}_{y y}[t-1]+(1-\lambda) \mathbf{y}[t] \mathbf{y}^{H}[t],
$$

where $\mathbf{y}[t]$ denotes the sample of the multi-channel signal $\mathbf{y}$ at time $t$. The forgetting factor $\lambda$ is chosen close to 1 to obtain long-term estimates that mainly capture the spatial coherence between the microphone signals. In the $\mathrm{DANSE}_{K}$ algorithm, however, the statistics of the input signal $\tilde{\mathbf{y}}_{k}$ in node $k$, defined by (14), change whenever a node $q \neq k$ updates its filters, since some of the channels in $\tilde{y}_{k}$ are indeed outputs from a filter in node $q$. Therefore, when node $q$ updates its filters, parts of the estimated correlation matrices $\widetilde{\mathbf{R}}_{y y, k}$ and $\widetilde{\mathbf{R}}_{x x, k}, \forall k \in\{1, \ldots, J\} \backslash\{q\}$, may become invalid. Therefore, strategy (35) may not work well, since every new estimate of the correlation matrix then relies on previous estimates. Instead, either downdating strategies should be considered, or the correlation matrices have to be completely recomputed.

\section{Conclusions}

The simulation results described in this paper demonstrate that noise reduction performance in hearing aids may be significantly improved when external acoustic sensor nodes are added to the estimation process. Moreover, these simulation results provide a proof-of-concept for applying DANSE $_{K}$ in cooperative acoustic sensor networks for distributed noise reduction applications, such as in hearing aids. A more robust version of $\mathrm{DANSE}_{K}$, referred to as R-DANSE ${ }_{K}$, has been introduced and convergence has been proven. Batchmode experiments showed that R-DANSE ${ }_{K}$ significantly outperforms DANSE $_{K}$. The occurrence of limit cycles and the effectiveness of relaxation in the simultaneous updating procedure has been illustrated. Additional tests have been performed to quantify the influence of several parameters, such as the DFT size and TDOA's or delays within the communication link.

\section{Acknowledgments}

This research work was carried out at the ESAT laboratory of Katholieke Universiteit Leuven, in the frame of the Belgian Programme on Interuniversity Attraction Poles, initiated by the Belgian Federal Science Policy Office IUAP P6/04 (DYSCO, "Dynamical systems, control and optimization", 2007-2011), the Concerted Research Action GOA-AMBioRICS, and Research Project FWO no. G.0600.08 ("Signal processing and network design for wireless acoustic sensor networks"). The scientific responsibility is assumed by its authors. The authors would like to thank the anonymous reviewers for their helpful comments.

\section{References}

[1] H. Dillon, Hearing Aids, Boomerang Press, Turramurra, Australia, 2001.

[2] B. Kollmeier, J. Peissig, and V. Hohmann, "Real-time multiband dynamic compression and noise reduction for binaural hearing aids," Journal of Rehabilitation Research and Development, vol. 30, no. 1, pp. 82-94, 1993.

[3] J. G. Desloge, W. M. Rabinowitz, and P. M. Zurek, "Microphone-array hearing aids with binaural output . I. Fixed-processing systems," IEEE Transactions on Speech and Audio Processing, vol. 5, no. 6, pp. 529-542, 1997.

[4] D. P. Welker, J. E. Greenberg, J. G. Desloge, and P. M. Zurek, "Microphone-array hearing aids with binaural output. II. 
A two-microphone adaptive system," IEEE Transactions on Speech and Audio Processing, vol. 5, no. 6, pp. 543-551, 1997.

[5] I. L. D. M. Merks, M. M. Boone, and A. J. Berkhout, "Design of a broadside array for a binaural hearing aid," in Proceedings of IEEE Workshop on Applications of Signal Processing to Audio and Acoustics (WASPAA '97), October 1997.

[6] V. Hamacher, "Comparison of advanced monaural and binaural noise reduction algorithms for hearing AIDS," in Proceedings of IEEE International Conference on Acoustics, Speech and Signal Processing (ICASSP '02), vol. 4, pp. 40084011, May 2002.

[7] R. Nishimura, Y. Suzuki, and F. Asano, "A new adaptive binaural microphone array system using a weighted least squares algorithm," in Proceedings of IEEE International Conference on Acoustics, Speech and Signal Processing (ICASSP '02), vol. 2, pp. 1925-1928, May 2002.

[8] T. Wittkop and V. Hohmann, "Strategy-selective noise reduction for binaural digital hearing aids," Speech Communication, vol. 39, no. 1-2, pp. 111-138, 2003.

[9] M. E. Lockwood, D. L. Jones, R. C. Bilger, et al., "Performance of time- and frequency-domain binaural beamformers based on recorded signals from real rooms," The Journal of the Acoustical Society of America, vol. 115, no. 1, pp. 379-391, 2004.

[10] T. Lotter and P. Vary, "Dual-channel speech enhancement by superdirective beamforming," EURASIP Journal on Applied Signal Processing, vol. 2006, Article ID 63297, 14 pages, 2006.

[11] O. Roy and M. Vetterli, "Rate-constrained beamforming for collaborating hearing aids," in Proceedings of IEEE International Symposium on Information Theory (ISIT '06), pp. 2809 2813, July 2006.

[12] S. Doclo and M. Moonen, "GSVD-based optimal filtering for single and multimicrophone speech enhancement," IEEE Transactions on Signal Processing, vol. 50, no. 9, pp. 2230-2244, 2002.

[13] A. Bertrand and M. Moonen, "Distributed adaptive nodespecific signal estimation in fully connected sensor networks-Part I: sequential node updating," Internal Report, Katholieke Universiteit Leuven, ESAT/SCD, Leuven-Heverlee, Belgium, 2009.

[14] A. Bertrand and M. Moonen, "Distributed adaptive estimation of correlated node-specific signals in a fully connected sensor network," in Proceedings of IEEE International Conference on Acoustics, Speech and Signal Processing (ICASSP '09), pp. 2053-2056, April 2009.

[15] T. J. Klasen, T. Van den Bogaert, M. Moonen, and J. Wouters, "Binaural noise reduction algorithms for hearing aids that preserve interaural time delay cues," IEEE Transactions on Signal Processing, vol. 55, no. 4, pp. 1579-1585, 2007.

[16] S. Doclo, R. Dong, T. J. Klasen, J. Wouters, S. Haykin, and M. Moonen, "Extension of the multi-channel wiener filter with ITD cues for noise reduction in binaural hearing aids," in Proceedings of the International Workshop on Acoustic Echo and Noise Control (IWAENC '05), pp. 221-224, September 2005.

[17] S. Doclo, T. J. Klasen, T. Van den Bogaert, J. Wouters, and M. Moonen, "Theoretical analysis of binaural cue preservation using multi-channel Wiener filtering and interaural transfer functions," in Proceedings of the International Workshop on Acoustic Echo and Noise Control (IWAENC '06), September 2006.

[18] T. Van den Bogaert, J. Wouters, S. Doclo, and M. Moonen, "Binaural cue preservation for hearing aids using an interaural transfer function multichannel wiener filter," in Proceedings of
IEEE International Conference on Acoustics, Speech and Signal Processing (ICASSP '07), vol. 4, pp. 565-568, April 2007.

[19] S. Doclo, M. Moonen, T. Van den Bogaert, and J. Wouters, "Reduced-bandwidth and distributed MWF-based noise reduction algorithms for binaural hearing aids," IEEE Transactions on Audio, Speech, and Language Processing, vol. 17, no. 1, pp. 38-51, 2009.

[20] A. Bertrand and M. Moonen, "Distributed adaptive node-specific signal estimation in fully connected sensor networks-Part II: simultaneous \& asynchronous node updating," Internal Report, Katholieke Universiteit Leuven, ESAT/SCD, Leuven-Heverlee, Belgium, 2009.

[21] J. B. Allen and D. A. Berkley, "Image method for efficiently simulating small-room acoustics," The Journal of the Acoustical Society of America, vol. 65, no. 4, pp. 943-950, 1979.

[22] M. Nilsson, S. D. Soli, and J. A. Sullivan, "Development of the hearing in noise test for the measurement of speech reception thresholds in quiet and in noise," The Journal of the Acoustical Society of America, vol. 95, no. 2, pp. 1085-1099, 1994.

[23] J. A. Bondy and U. S. R. Murty, Graph Theory with Applications, American Elsevier, New York, NY, USA.

[24] S. Maraboina, D. Kolossa, P. K. Bora, and R. Orglmeister, "Multi-speaker voice activity detection using ICA and beampattern analysis," in Proceedings of the European Signal Processing Conference (EUSIPCO '06), 2006.

[25] V. Berisha, H. Kwon, and A. Spanias, "Real-time implementation of a distributed voice activity detector," in Proceedings of IEEE Sensor Array and Multichannel Signal Processing Workshop (SAM '06), pp. 659-662, July 2006. 